\title{
MANAGEMENT OF DISTAL TIBIAL INTRA-ARTICULAR FRACTURES [TIBIAL PLAFOND] BY VARIOUS METHODS: A PROSPECTIVE STUDY
}

\author{
K. Nagamuneendrudu1, Mettu Srirangarao ${ }^{2}$, Harsha Bogadhi ${ }^{3}$ \\ ${ }^{1}$ Associate Professor, Department of Orthopaedics, Osmania General Hospital/College, Hyderabad. \\ ${ }^{2}$ Assistant Professor, Department of Orthopaedics, Osmania General Hospital/College, Hyderabad. \\ ${ }_{3}^{3}$ Postgraduate, Department of Orthopaedics, Osmania General Hospital/College, Hyderabad.
}

\begin{tabular}{l}
\hline ABSTRACT \\
AIM \\
To evaluate the results of various methods of management of Tibial Pilon Fractures. The purpose of the present study is to verify \\
the functional outcome of various operative techniques in terms of efficacy, complications and to rationalise the indications for the \\
various procedures.
\end{tabular}

\section{MATERIALS AND METHODS}

The study included 25 patients reporting to Osmania General Hospital, Hyderabad, from November 2012 to February 2014.

\section{RESULT}

The age is between 10-60 yrs. majority in the age group of 20-40 yrs. (60\%) with male predominance in 18 cases $(72 \%)$, right side is common in 15 cases (60\%), high velocity injuries are majority 16 (64\%), open fractures are 17 (68\%). Axial compression and shear is the common mode of injury 12 (48\%) followed by axial compression 8 (32\%). Excellent result in 2 cases (8\%), Good result in 12 cases (48\%), Fair result in 1 case (4\%) and Poor result in 10 cases (40\%).

\section{CONCLUSION}

Tibial pilon fractures are more common in males of $3^{\text {rd }}$ and $4^{\text {th }}$ decade, productive age with socio-economic consequences. Most high velocity fractures are Grade III B compound fractures. Axial compression in fall from height and axial compression with shear in road traffic cases is the most common type of mechanism of injury. Low velocity pilon fractures common in female and osteoporotic patients. Fracture comminution, soft tissue problems are the main factors deciding the outcome of the management. Early anatomical reduction, a stable fixation, early mobilisation and delayed weight-bearing seem to improve long-term results of treatment in Pilon fractures caused by high energy trauma. Ilizarov external skeletal fixation and minimal internal fixation of distal tibial pilon fractures is a good method for treating all types of intra-articular pilon fracture. MIPPO in selected cases gave best results.

\section{KEYWORDS}

Pilon Fractures, Ilizarov Fixator, MIPPO Minimally Invasive Plate Osteosynthesis.

HOW TO CITE THIS ARTICLE: Nagamuneendrudu K, Srirangarao M, Bogadhi H. Management of distal tibial intra-articular fractures [tibial plafond] by various methods - a prospective study. J. Evolution Med. Dent. Sci. 2016;5(70):5081-5085,

DOI: $10.14260 /$ jemds/2016/1154

\section{INTRODUCTION}

The distal tibial intra-articular fractures are also called as Plafond fractures. These fractures are caused by axial forces and rotational violence, mostly due to fall from height, motor vehicle accidents. These fractures are caused by the dome of talus being driven into the tibia plafond, as the talus dome like a pestle is driven into the distal tibia through the ankle joint called as Pilon Fracture name coined by French radiologist, Etienne Destot. This fracture involves dome of the distal tibial articular surface and extends into the adjacent metaphysis. The fibula may or may not be intact, Pilon fractures are rare comprising $3-10 \%$ of all tibial fractures and are notorious for their morbidity due to the increased comminution of the articular surface and of the metaphysic of the distal tibia, lack of adequate soft tissue cover to the

Financial or Other, Competing Interest: None.

Submission 23-06-2016, Peer Review 29-07-2016,

Acceptance 25-07-2016, Published 01-09-2016.

Corresponding Author:

Mettu Srirangarao,

H. No. 12-13-826/2A STR No. 11,

Keemthi Colony,

Hyderabad-500017.

E-mail:mettu2000@gmail.com

DOI: $10.14260 /$ jemds/2016/1154 distal tibia, most often results in either soft tissue swelling or compound fractures that result in deformity, stiffness and chronic pain, secondary osteoarthritis, management of these fractures is a challenge. These fractures continuously are being studied to evolve treatment strategies. Ruedi and Allgower ${ }^{1,2,3}$ classified these fractures and concluded that anatomical reduction and stable fixation is needed to be achieved provided good surgical technique and careful handling of soft tissue are done. Bone LB ${ }^{4}$ concluded that best results have occurred when stable internal fixation is accomplished using plates and screws. Basic steps in reconstruction are restoration of anatomic length of the fibula with plate fixation, reconstruction of the tibial articular surface, cancellous bone grafting of the metaphyseal defect and buttress plating either anteriorly or medially of the distal tibia.

The most common classification of the soft tissue injury in current use is that of Tscherne and Goetzen. 5 Treatment strategies and fixation methods used include internal and external fixation as well as staged approaches with aim of reducing the fracture, reconstructing the involved bones and restoration of articular surface congruence with minimal insult to soft tissues. Appropriate wound management is important to reduce high rate of wound healing and infectious 
complications with open Pilon fractures, care should be taken by pre-operative planning and post-operative of rehabilitation for best results.

\section{MATERIALS AND METHODS}

All the cases taken for study were those attending Osmania General Hospital during the period of November 2012 to February 2014. The following protocol was adopted for evaluation of the cases.

\section{Inclusion Criteria}

1. Young and active patients.

2. All types of pilon fractures.

3. All closed and open fractures.

\section{Exclusion Criteria}

1. Very elderly patients

2. Patients who cannot follow rehabilitation programmes.

3. Chronic smokers and alcoholics.

4. Patients with severe osteoporosis.

\section{Treatment Protocol}

1. Undisplaced fractures, certain minimally displaced type A fractures or type $\mathrm{C} 1$ fractures may be treated by a long leg cast for 6 weeks followed by functional cast, brace or treated in an external fixation without reduction followed by ROM exercises. When treatment without reduction is chosen, the time of weight bearing and the timing of removal of external immobilisation must be individualised depending on the fracture.

2. Displaced fracture were treated surgically with various modalities (Open reduction and internal fixation with plate, two-stage delayed open reduction and internal fixation, combined external and limited internal fixation and external fixation and fibula plating, Ilizarov external fixation.

The patients are screened thoroughly for fitness for surgery, X-ray of ankle mortise view, lateral view whenever required in suspected diaphyseal extension X-ray of the entire leg is taken. CT scan is done for detailed study of anatomy of fracture.

\section{Initial Management}

1. Long leg splint: The fracture should be immobilised and elevated by placing the ankle in a calcaneal pin traction and to elevate the limb on a Bohler frame. It is usually enough to maintain the talus in a provisionally reduced position. This allows easy assessment of the skin and soft tissues, maintains elevation and provisionally reduces the talus. The fracture is definitively treated after planning and when the condition of the soft tissues is satisfactory.

2. Spanning fixator, the frames are frequently constructed from simple pin-bar components. This procedure is carried out in emergency and it is used as a means to maintain length and provisionally align the fracture before definitive treatment. This technique is an important component of protocols in long surgical delays before definitive surgery and helps to the decreased soft tissue complications. In patients with multiple injuries, open wounds or compartment syndrome, a provisional spanning external fixator should routinely be applied.

\section{Definitive Management}

Patients return to the operating room for a second procedure for definitive reduction and fixation of the articular surface when soft tissue swelling has subsided usually within 10-14 days. Patterson and Cole et $\mathrm{al}^{6}$ described immediate fibular fixation and placement of a medial spanning external fixator, followed an average of 24 days later by removal of the fixator and open reduction and internal fixation.

Contoured Locking Plates and Screws when the Soft Tissues are not Injured Definitive Plating is done with Contoured Locking Plate and Screws
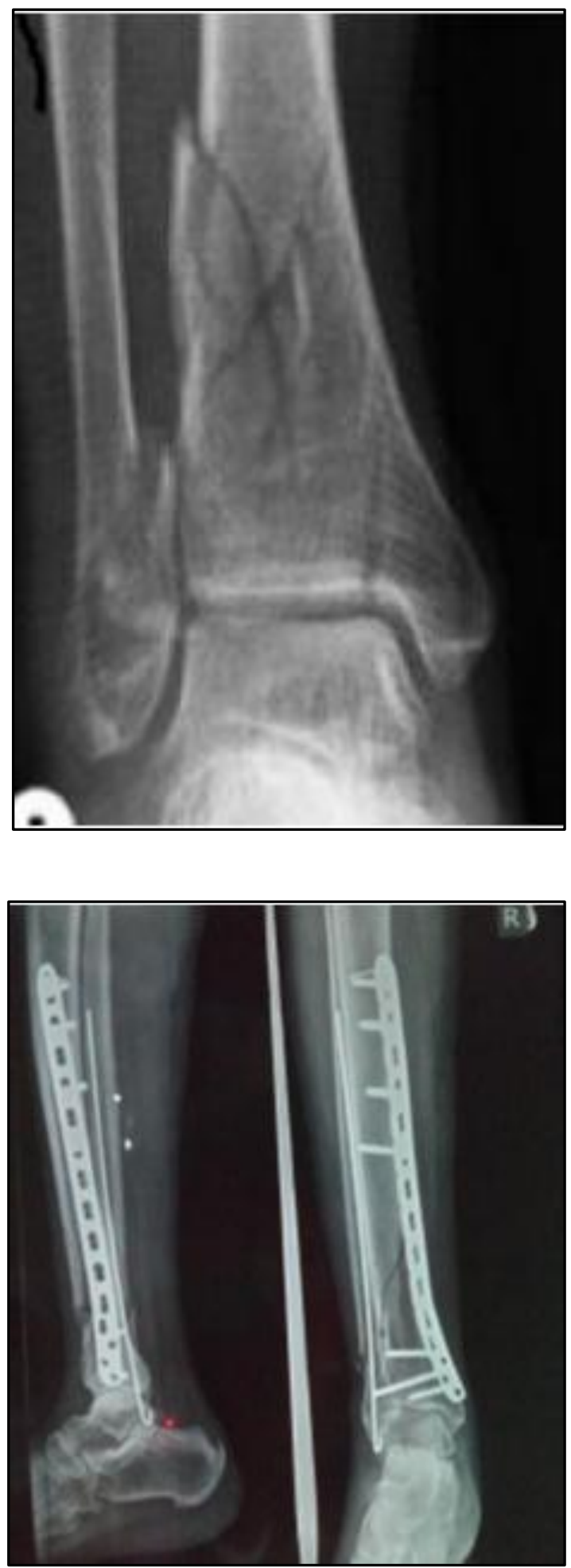
Ilizarov Ring Fixator or Hybrid Wire Fixator is used. When the Soft Tissue is Intact, but Not Healthy
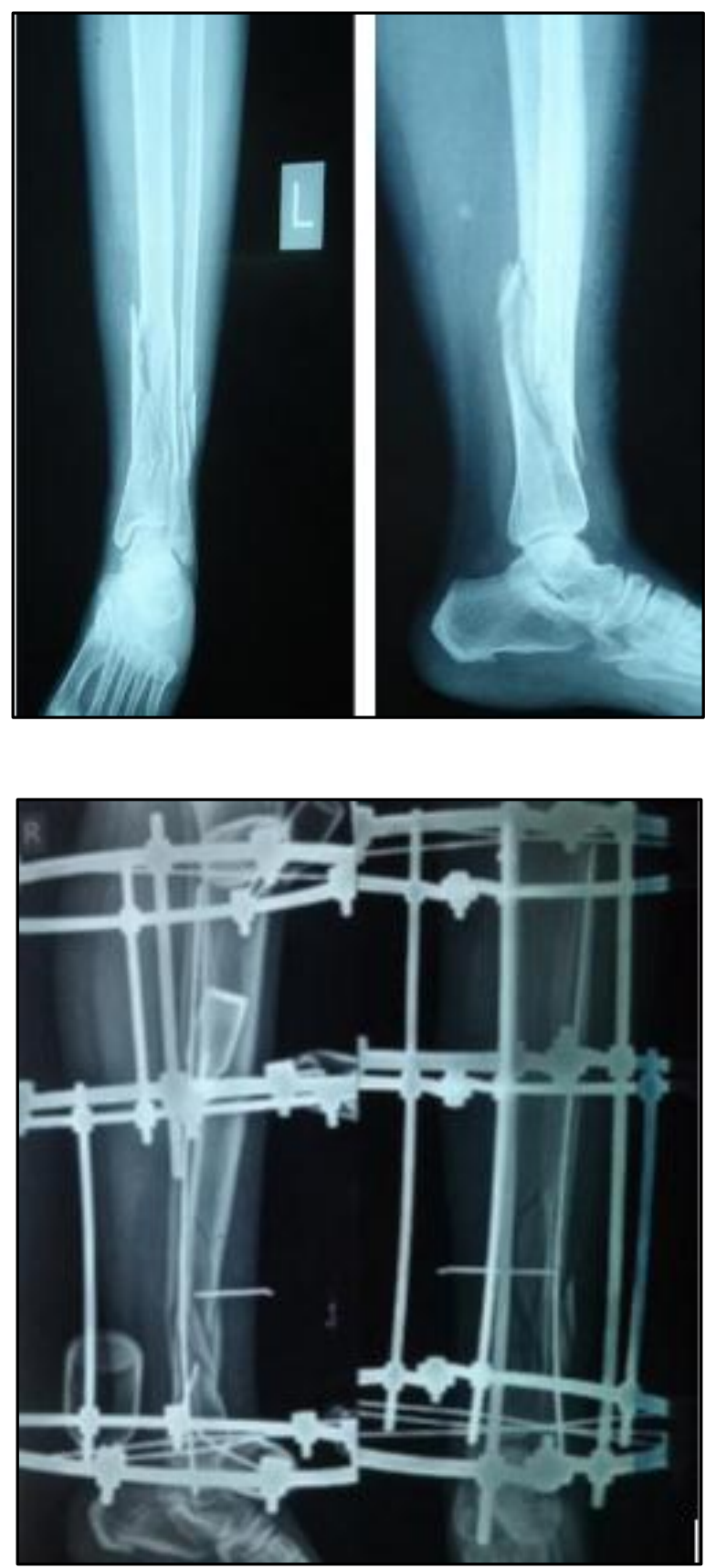

Spanning External Fixator when there is Wound Requiring Wound Management, Soft Tissue Cover, is applied

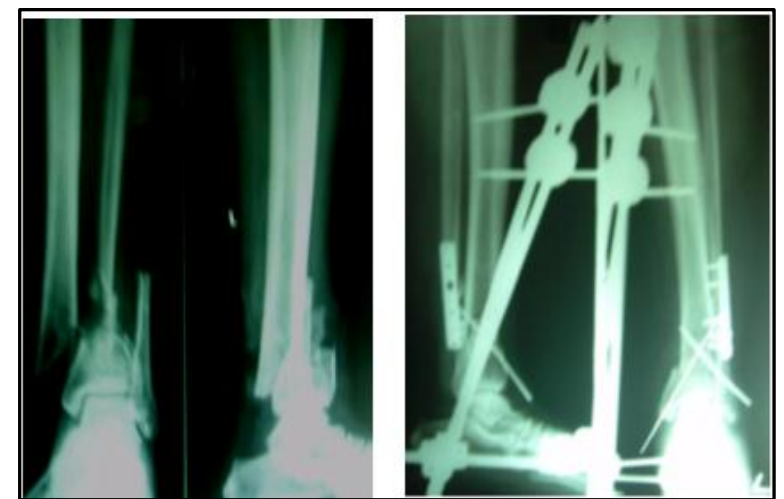

The approach is antero-medial or antero-lateral mostly minimally invasive, posterior lateral for fixation of fibula and plafond. Percutaneous for limited fracture fixation along with wire or external fixator. Primary arthrodesis has been suggested as a method of treating severely comminuted tibial Pilon fractures.

Primary arthrodesis may be considered for severe open injuries with extensive loss of cartilage from the tibial and talar articular surfaces. The wound is debrided and the remaining cartilage is removed from the talus and tibia. An external fixator can be used to stabilise the fracture. Bone grafting may be necessary when the soft tissues have healed. In these severe, open injuries, functional results often are poor. Amputation may be an alternative.

\section{THE RESULTS ARE EVALUATED USING BURWELL AND CHARNLEY CRITERIA (1965). ${ }^{7}$ \\ OBJECTIVE CRITERIA}

Good: Ankle movements at least three quarters of normal. Trivial swelling. Normal gait.

Fair: Ankle movements at least half of normal. Moderate swelling. Normal gait.

Poor: Ankle movements less than half of the normal. Severe swelling with visible deformity of ankle. Patient walking with limp.

\section{SUBJECTIVE CRITERIA}

All patients were questioned as to the presence of pain, sensation of stiffness and any disability. The grading is given below:

Good: Complete recovery.

Fair: Aching during use. Slight stiffness is present. Ability to walk is not seriously impaired.

Poor: Serious impairment of ability to walk or work.

\section{RESULTS}

The age is between 10-60 yrs. majority in the age group of 2040 yrs. (60\%). No age group is immune. It is most common in third and fourth decades of life. In children, pilon fractures were not seen. They presented as epiphyseal injuries, of which Salter-Harris type II was most common followed by type 1 . Male predominance in 18 cases (72\%) 3 times than females. There is little difference between right and left side incidence. Right side is common in 15 cases $(60 \%)$, high velocity injuries are majority 16 (64\%), open fractures are 17 (68\%). Axial compression and shear is the common mode of injury 12 (48\%) followed by axial compression $8(32 \%)$. Type 1 are seven cases (28\%), type 2 eleven cases (44\%), type 3 eight cases (32\%). Excellent result in 2 cases $(8 \%)$, Good result in 12 cases (48\%), Fair result in 1 case (4\%), Poor result in 10 cases $(40 \%)$.

\section{DISCUSSION}

Most of the patients in present study were from age group of 3 rd to 4 th decade of life. The average age in this study was 38.2 years. This is significantly lower compared to that quoted by other authors in literature, Koulouvaris $\mathrm{P}$ et al 48.4 yrs. and Vidyadhara et al 34.5 yrs. Our results are comparable with Indian study of Vidyadhara et al with age distribution of 28-52 yrs. A significant male preponderance of $72 \%$ was noted in this study as compared to the female preponderance, Koulouvaris $\mathrm{P}$ et al $74 \%$ and Vidyadhara et al $80 \%$ years. High velocity injuries like road traffic accidents and fall from 
heights accounted for $70 \%$ of these fractures and most of them were males. In $30 \%$ of patients, fracture is a result of trivial fall and majority of them are elderly age group patients, especially females; $60 \%$ of the fractures involved on the right side in present study as compared to $58 \%$, Vidhyadhara et al and $40 \%$ involved the left as compared to $42 \%$ in Vidhyadhara et al. Majority fractures are type III eight cases (32\%), type II eleven cases (44\%), type 1 are seven cases (28\%). The incidence of open tibial pilon fractures were more common $(64 \%)$ than closed fractures, since majority were high velocity injuries. Axial compression injuries were 32\% and combined axial compression and shear were $48 \%$. Combined axial compression and shear type injury due to fall from a height and RTA was the most common type of injury. Closed Displaced Pilon fractures were fixed with minimally invasive plating with small incision. All had good-to-excellent results. Müller ${ }^{8}$ obtained excellent results in $82 \%$ of cases treated with minimally invasive plating and one case developed non-union. However, we had no non-union in our series.

Grade II and III type of compound Pilon fractures were treated with debridement and stabilised with external fixation with unilateral external fixators or Ilizarov in all these cases with stabilisation of fibular fracture wherever necessary. In these cases infection rate and malunion noted. In Type I fractures are in 3-4th decade, treated conservatively with good results, Type II fractures are also in 3-4th decade treated with ORIF, External fixation, Ilizarov, had good results in majority of cases (82\%), cases treated with Ilizarov showed consistently good results. Type III fractures majority are compound fractures (70\%), treated with external fixator, the remaining $(30 \%)$ presented with soft tissue injury with gross swelling, treated with Ilizarov fixator, irrespective mode of treatment the results are consistently poor.

In Type III developed post-traumatic arthritics due to mal-alignment of the joint. These cases are being followed up with physiotherapy and mild analgesics. Even though, Thordarson $\mathrm{DB},{ }^{9}$ advocated primary fusion of the ankle in

their article we have not attempted any primary fusion.

The deformities observed are mostly posterior and lateral angulation of the fracture, and Tibial shortening of $1 \mathrm{~cm}$ mainly due to fracture comminution, metaphyseal impaction resulting in mal union.

Understanding the mechanism causing tibial plafond fracture is of major importance in order to choose the optimal method of treatment. The differences regarding the treatment principles between fractures caused by axial compression and those caused by torsional forces.

The stability of the ankle joint is not enhanced by fibula fixation, because axial compression fractures are not accompanied by ligamentous damage. $1,2,3$ If we reconsider that the major stabilising element of the ankle joint is the deltoid ligament at the medial side, we can conclude that reduction and fixation of the fibula in such fractures has no significant effect in the stability of the ankle joint.

In fractures caused by torsional forces, the articular surface is usually easier to reconstruct by internal fixation. ${ }^{10}$ In this case, ankle instability, major concern in these fractures should be the restoration of the stability of the ankle joint by repair of the ligamentous elements. ${ }^{11}$ Essential goal is to restore all structures needed in order to achieve optimal talus movement in relation to the tibia. Although axial compression, rotational forces are largely responsible, Kellam ${ }^{12}$ drew attention to the significant counterforce from the talus which damages the articular surface of the tibia to a greater extent than can be appreciated radiologically. Boerner ${ }^{13}$ found fractures of the talus in 3 percent of his patients, while Kellam and Waddell 13 reported splits in the articular cartilage of the dome of the talus in all their cases treated by internal fixation. There were no associated fractures of the talus in this series. Open reduction and internal fixation is indicated in types II and III Pilon fractures. Good reduction is generally rewarded by good functional results. Non-operative management achieves good results in type I fractures; however, the good position may be lost in the plaster. Hind foot function is negligibly affected in type I fractures. Internal fixation is followed by recovery of hind foot function in types II and III pilon fractures.

Ruedi et al1,2,3 reported 74-90\% good or excellent results in their series after open reduction and internal fixation. Most of the fractures in their series were of a low energy pattern. Mast et $\mathrm{al}^{14}$ using the same technique reported $78 \%$ good results on 37 patients with a minimum follow-up of 6 months. Grose et al also reported good-to-excellent results. On the other hand, a number of authors reported dismal results with open reduction and internal fixation. Bourne ${ }^{13}$ reported on 16 AO type C fractures with only $44 \%$ satisfactory results. Müller et $\mathrm{al}^{9}$ had $40 \%$ complication. Teeny and Wiss ${ }^{15}$ reported $50 \%$ poor results of 60 Pilon fractures with open reduction and internal fixation, the high complication rate with open reduction and internal fixation especially in high energy fractures can be diminished with careful selection of patients, taking into consideration the soft tissues injury, degree of comminution, intra-articular extension, prompted the use of less invasive techniques such as limited open reduction and internal fixation and hybrid fixators. Bone, 4 Tornetta $3 r d$ et al16, Ristiniemi et al ${ }^{17}$ Pugh et al ${ }^{18}$ In a study by Watson et al ${ }^{19}$ in a randomised study Pilon Fractures were treated with either open reduction and internal fixation or external fixation with limited internal fixation. After a 2-year followup, the major complications were much greater in the group of open reduction and internal fixation, whereas the clinical and radiographic outcomes of the two groups were the same. The above report is strongly indicative for the use of external fixation in high energy Pilon fractures, our study also recommends the external fixation for high energy fractures.

The application of Ilizarov external fixations as a definite treatment for Pilon fractures has radically changed their prognosis. ${ }^{19}$ By minimising soft tissue dissection, decreases infection rate and permits early mobilisation of the ankle joint through hinges in a stable mechanical environment. ${ }^{1}$ The first step before the application of the external fixation is anatomical reduction of the articular surface, achieved with a small skin incision. Reduced and fixed anatomically by olive wires adjusted properly to the external fixator. The use of internal fixation is rarely required, while the use of bone grafts is very limited. Fixation of the fibula in fractures caused by axial compression, which are treated by external fixation is not indicated. Anatomical reduction of the fibula does not allow fragment contact at the distal tibia metaphysis and has been associated with high incidence of delayed union or pseudarthrosis. ${ }^{3}$

Early mobilisation of the ankle joint is another advantage of the Ilizarov device.20,21 In fractures caused by axial 
compression and no concomitant ligamentous instability, best results can be achieved by early mobilisation and partial weight bearing with fixator in situ.

\section{CONCLUSIONS}

1. Tibial pilon fractures are more common in males of 3 rd and $4^{\text {th }}$ decade.

2. Axial compression is the most common type of mechanism of injury.

3. Most Tibial pilon fractures are high velocity Grade III B compound fractures.

4. Low impact pilon fractures are common in Female and osteoporotic patients.

5. Early anatomical reduction, a stable fixation, early mobilisation, and delayed weight-bearing seem to improve long-term results of treatment in pilon fractures caused by high energy trauma.

6. Ilizarov external skeletal fixation and minimal internal fixation of distal tibial pilon fractures is a good method for treating all types of intra-articular pilon fracture.

7. Minimally invasive plating in selected cases gave best results.

\section{REFERENCES}

1. Ruedi T. Fractures of the lower end of the tibia into the ankle joint: results 9 years after open reduction and internal fixation. Injury 1973;5(2):130-4.

2. Ruedi TP, Allgower M. The operative treatment of intraarticular fractures of the lower end of the tibia. Clin Orthop 1979;138:105-10.

3. Ruedi T, Allgower M. Fractures of the lower end of the tibia into the ankle joint. Injury 1969;1(2):92-9.

4. Bone LB. Fractures of the tibial plafond. The pilon fracture. The Orthopedic Clinics of North America 1987;18(1):95-104.

5. Tscherne HG, Gotzen L. Fractures with soft tissue injuries. Berlin: Springer-Verlag, 1984.

6. Patterson MJ, Cole JD. Two-staged delayed open reduction and internal fixation of severe pilon fractures. Journal of Orthopaedic Trauma 1999;13(2):85-91.

7. Burwell HN, Charnley AD. The treatment of displaced fractures at the ankle by rigid internal fixation and early joint movement. J Bone Joint Surg 1965;47(4):634-60.

8. Müller FJ, Nerlich M. Tibial pilon fractures. Acta Chir Orthop Traumatol Cech 2010;77(4):266-76.
9. Thordarson DB, Motamed S, Hedman T, et al. The effect of fibular malreduction on contact pressures in an ankle fracture malunion model. J Bone Joint Surg Am 1997;79(12):1809-15.

10. Egol KA, Wolinsky P, Koval KJ. Open reduction and internal fixation of tibial pilon fractures. Foot and Ankle Clinics 2000;5(4):873-85.

11. Giaclino AA, Hammond DI. The relationship between oblique fractures of the medial malleolus and concomitant fractures of the anterolateral aspect of the tibial plafond. JBJS 1987;69(3):381-4.

12. Kellam JF, Waddell JP. Fractures of the distal tibial metaphysis with intra-articular extension - the distal tibial explosion fracture. J Trauma 1979;19(8):593-601.

13. Bourne RB, Rorabeck, Macnab JBA. Intra-articular fractures of the distal tibia: the pilon fracture. Journal of Trauma-Injury Infection \& Critical Care 1983;23(7).

14. Jeffrey MW, Phillip SG, Pappas JIM. Fractures of the tibial pilon. Clinical Orthopaedics \& Related Research 1988; Vol 230.

15. Teeny SM, Wiss DA. Open reduction and internal fixation of tibial plafond fractures: variables contributing to poor results and complications. Clin Orthop Relat Res 1993;292:108-17.

16. Paul T, Lon W, Marc B, et al. Pilon fractures: treatment with combined internal and external fixation. Journal of Orthopaedic Trauma 1993;7(6):489-96.

17. Ristiniemi J, Flinkkilä T, Hyvönen $\mathrm{P}$, et al. Two-ring hybrid external fixation of distal tibial fractures: a review of 47 cases. Journal of Trauma 2007;62(1):174-83.

18. Kevin PJ, Philip WR, McAndrew, et al. Tibial pilon fractures: a comparison of treatment methods. Journal of Trauma-Injury Infection \& Critical Care 1999;47(5):937.

19. Watson JT, Moed BR, Karges DE, et al. Treatment protocol based on severity of soft tissue injury. Clinical Orthopaedics \& Related Research 2000;375:78-90.

20. Vasiliadis ES, Grivas TB, Psarakis SA, et al. Advantages of the Ilizarov external fixation in the management of intraarticular fractures of the distal tibia. Journal of Orthopaedic Surgery and Research 2009;4:35. doi:10.1186/1749-799X-4-35.

21. McDonald, Mark G, Ronald BC, et al. Ilizarov treatment of pilon fractures. Clinical Orthopaedics \& Related Research 1996;325:232-8. 